\title{
A combined numerical and experimental framework for determining permeability properties of the arterial media
}

\author{
A. Comerford · K.Y Chooi • M. Nowak • P. D. Weinberg • S. J. Sherwin
}

Received: date / Accepted: date

\begin{abstract}
The medial layer of the arterial wall may play an important role in the regulation of water and solute transport across the wall. In particular, a high medial resistance to transport could cause accumulation of lipid carrying molecules in the inner wall. In this study, the water transport properties of medial tissue were characterised in a numerical model utilising experimentally obtained data for the medial microstructure and the relative permeability of different constituents. For the model, a new solver for flow in porous materials, based on a high-order splitting scheme, was implemented in the spectral/hp element library nektar ++ and validated. The data were obtained by immersing excised aortic bifurcations in a solution of fluorescent protein tracer and subsequently imaging them with a confocal microscope. Cuboidal regions of interest were selected in which the microstructure and relative permeability of different structures were transformed to a computational mesh. Impermeable objects were treated fictitiously in the numerical scheme. On this cube, a pressure drop was applied in the three coordinate directions and the principal components of the permeability tensor were determined. The reconstructed images demonstrated the arrangement of elastic lamellae and interspersed smooth muscle cells in rat aortic media; the distribution and alignment of the smooth muscle cells varied spatially within the extracellular matrix. The numerical simulations high-
\end{abstract}

A. Comerford · M. Nowak · S. J. Sherwin

Department of Aeronautics, Imperial College London, South Kensington Campus, SW7 2AZ, London, UK E-mail: a.comerford@imperial.ac.uk

K.Y Chooi · P. D. Weinberg

Department of Bioengineering, Imperial College London, South Kensington Campus, SW7 2AZ, London, UK lighted that the heterogeneity of the medial structure is important in determining local water transport properties of the tissue, resulting in regional and directional variation of the permeability tensor. A major factor in this variation is the alignment and density of smooth muscle cells in the media, particularly adjacent to the adventitial layer.

\section{Introduction}

Atherosclerosis is the leading cause of morbidity and mortality in the developed world. The disease is characterised by a build up of lipid, inflammatory cells and fibrous proteins in the intima and inner media of the arterial wall. The precise mechanisms involved in the development of the disease, especially in its early stages, are still disputed, but the uptake of lipid-carrying plasma macromolecules is thought to play a key role (Nielsen, 1996, Weinberg, 2004, Tarbell, 2003, 2010); hence, studies of the rate of entry and transport within the wall of macromolecules could increase our understanding of the disease. Water transport, the subject of this paper, is important because much macromolecule transport is convective (A.Tedgui and Lever, 1985) (The Peclet number for LDL based on mean smooth muscle cell radius is 4.6 (Huang and Tarbell, 1997, Tada and Tarbell, 2004)).

Transport of water within the media is of interest as macromolecules may accumulate in the intima not because they enter the wall more rapidly but because, once they have entered the intima, they leave more slowly across the media and into the adventitia (see Tarbell (2003) and references therein). The medial layer consists of smooth muscle cells (SMCs), water, glycosaminoglycans and structural molecules - principally collagen and elastin 
(Parker and Winlove, 1988). These components are distributed in the media in a repeating architecture including aligned elastic lamellae (Clark and Glagov, 1985). Water and dissolved solutes crossing the media do not have equal access to all these compartments; they are excluded by cells and partially excluded, to differing extents, by the other components. This restriction may inhibit transport across the layer, leading to accumulation of macromolecules in the inner wall. Transport properties of the medial layer are also important in understanding the transport of drugs from stents (Vairo et al., 2010, Denny et al., 2012, Cutri et al., 2013, Bozsak et al., 2014).

Transport of water and LDL in the arterial wall has received considerable attention both in studies utilising simple 2D \& 3D models of the media (Wang and Tarbell, 1995, Huang and Tarbell, 1997, Tada and Tarbell, 2000, 2002, Dabagh et al., 2009a) as well as in models of the whole arterial wall (Prosi et al., 2005, Ai and Vafai, 2006, Sun et al., 2009, Dabagh et al., 2009b). In the former studies, the medial layer was treated as an array of circles representing SMCs, embedded in the ground substance (GS). In the latter studies, the media was treated as a homogenous layer in which the properties were measures of effective permeability obtained from the former studies or studies derived from these works. One drawback of these methods is that the geometry is either completely or partially homogenous; another is that the influence of the elastic lamellae is ignored. Hence reliable information about medial transport details remain elusive. This issue has previously been highlighted by Khakpour and Vafai (2008). Experimentally, local transport properties are extremely difficult if not impossible to obtain; however, the properties on which they depend and which are required for better models can be reliably assessed. Ultimately, with the development of an advanced model, driven by experimental data that include the cellular make up of the medial layer, it will be possible to determine potential sites of protein accumulation in the different wall layers and the effects of changing wall properties (e.g. SMC tone) on such accumulation.

The present study aims to characterise water transport in the medial layer and to determine how the medial structure hinders such transport. This is achieved through a number of steps. Firstly, a solver for Brinkman's equation is developed and validated against known benchmark solutions. Secondly, the solver is used to model transport in porous media using previously developed solutions for the arterial media. Finally, a novel strategy, combining experimental data and numerical modelling, is introduced and used to determine the permeability of the arterial media.

\section{Unsteady Brinkman's equation}

Flow in porous materials that include regions void of fluid, such as the arterial media, can be described by Brinkman's equation,

$\frac{\partial \mathbf{u}}{\partial t}=-\nabla p+\nu \nabla^{2} \mathbf{u}-\frac{\nu}{\overline{\overline{\boldsymbol{\kappa}}}} \mathbf{u}+\mathbf{f}, \quad$ in $\Omega^{B} \times[0, T]$

$\nabla \cdot \mathbf{u}=0 . \quad$ in $\Omega^{B} \times[0, T]$

where $\mathbf{u}$ is the velocity, $p$ the pressure, $\nu$ the effective viscosity, $\overline{\overline{\boldsymbol{\kappa}}}$ the Darcian permeability tensor and $\mathbf{f}$ a forcing term. Unlike Darcy's law, Brinkman's equation can characterise the flow around solid obstacles embedded in a porous material (Khaled and Vafai, 2003). In particular, the additional term $\nu \nabla^{2} \mathbf{u}$ accounts for the formation of boundary layers in the vicinity of solid obstacles. The equation has been widely used for modelling transport in the medial layer.

\subsection{Smooth muscle cell volume fraction}

As noted above, the medial layer of the arterial wall consists of cells, water, glycosaminoglycans and structural proteins. These together define a porous medium in which water is excluded from some areas. For example, mobile water is completely excluded from SMCs. The SMC volume fraction of the medial layer, $\phi$, is defined by

$\phi=\frac{V_{S M C}}{V_{\text {media }}}$

where $V_{S M C}$ is the volume of SMCs and $V_{\text {media }}$ is the volume of the whole medial region of interest.

\subsection{Effective permeability}

Effective permeability refers to the permeability of a hypothetical homogeneous region of tissue that exhibits the same fluid-mechanical properties as the heterogeneous structure, given the same hydraulic gradient. In the present context, heterogeneous refers to a porous structural medium with 
solid obstacles dispersed in it, whilst the homogenous representation is a single medium (i.e. one with no solid obstacles) having the effective properties of the original structure. Figure 1 illustrates this concept. The heterogeneous structure consists of a fluid (porous) domain and a solid domain, $\Omega^{B}=\Omega_{f}^{B} \cup \Omega_{s}^{B}$. The boundary of the solid domain $\partial \Omega_{s}^{B}$ (red in Figure 1) denotes the interface between solid and fluid domain, while the boundary of the fluid domain $\partial \Omega_{f}^{B}$ (blue in Figure 1) denotes the fluid boundaries. The dashed black square is the region over which the effective permeability is calculated. This approach is referred to as oversampling (Hou and Wu, 1997) and takes away the influence of boundary conditions. It is suitable for the present application since the boundary layer thickness is $O(\sqrt{\kappa})$ (Huang and Tarbell, 1997). This also means that the size of the dashed black region can be close to the size of the domain.

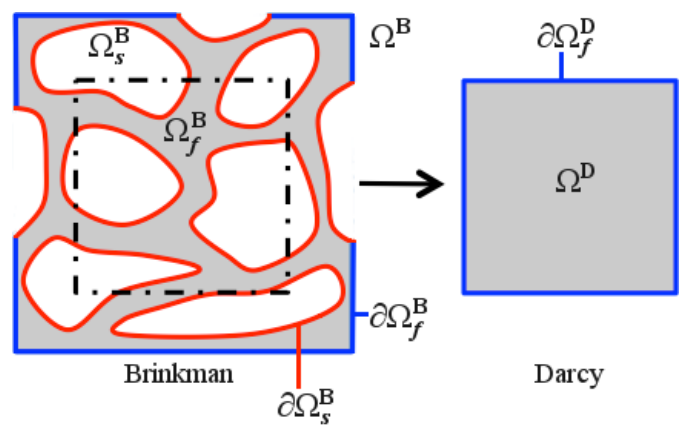

Fig. 1: Effective permeability of a block of porous material.

The dashed black region can be extracted from the domain and its effective permeability tensor can be calculated. In this homogenised region the flow is governed by Darcy's equation, given by:

$\langle\mathbf{u}\rangle=\frac{1}{\nu} \overline{\overline{\boldsymbol{\kappa}}}_{\mathrm{eff}}\langle\nabla p\rangle \quad$ in $\Omega^{D}$

in which $\langle\mathbf{u}\rangle$ donates the volume average velocity and $\overline{\boldsymbol{\kappa}}_{\text {eff }}$ the permeability tensor, which is symmetric (e.g. $\kappa_{r \theta}=\kappa_{\theta r}$ ); it is given by,

$\overline{\overline{\boldsymbol{\kappa}}}_{\mathrm{eff}}=\left[\begin{array}{ccc}\kappa_{r r} & \kappa_{r z} & \kappa_{r \theta} \\ \kappa_{r z} & \kappa_{z z} & \kappa_{z \theta} \\ \kappa_{r \theta} & \kappa_{z \theta} & \kappa_{\theta \theta}\end{array}\right]$

To calculate the full tensor, 3 numerical experiments must be performed in three coordinate directions $(r, z$ and $\theta$ represent the axes of a right- handed cylindrical coordinate system). In these experiments a pressure drop is applied to the domain and the resulting mean velocity field and pressure gradient are extracted. This leads to the following matrix equation when the permeability tensor is assumed to be symmetric:

$\mathbf{F} \cdot \mathbf{k}=\langle\mathbf{u}\rangle$

where $\mathbf{k}=\left[\begin{array}{c}\kappa_{r r} \\ \kappa_{z z} \\ \kappa_{\theta \theta} \\ \kappa_{r z} \\ \kappa_{r \theta} \\ \kappa_{z \theta}\end{array}\right]$ and $\langle\mathbf{u}\rangle=\left[\begin{array}{c}\langle\mathbf{u}\rangle^{1} \\ \langle\mathbf{v}\rangle^{1} \\ \langle\mathbf{w}\rangle^{1} \\ \langle\mathbf{u}\rangle^{2} \\ \langle\mathbf{v}\rangle^{2} \\ \langle\mathbf{w}\rangle^{2} \\ \langle\mathbf{u}\rangle^{3} \\ \langle\mathbf{v}\rangle^{3} \\ \langle\mathbf{w}\rangle^{3}\end{array}\right]$

$\mathbf{F}=\left[\begin{array}{cccccc}\langle\nabla p\rangle_{r}^{1} & 0 & 0 & \langle\nabla p\rangle_{z}^{1} & \langle\nabla p\rangle_{\theta}^{1} & 0 \\ 0 & \langle\nabla p\rangle_{z}^{1} & 0 & \langle\nabla p\rangle_{r}^{1} & 0 & \langle\nabla p\rangle_{\theta}^{1} \\ 0 & 0 & \langle\nabla p\rangle_{\theta}^{1} & 0 & \langle\nabla p\rangle_{r}^{1} & \langle\nabla p\rangle_{z}^{1} \\ \langle\nabla p\rangle_{r}^{2} & 0 & 0 & \langle\nabla p\rangle_{z}^{2} & \langle\nabla p\rangle_{\theta}^{2} & 0 \\ 0 & \langle\nabla p\rangle_{z}^{2} & 0 & \langle\nabla p\rangle_{r}^{2} & 0 & \langle\nabla p\rangle_{\theta}^{2} \\ 0 & 0 & \langle\nabla p\rangle_{\theta}^{2} & 0 & \langle\nabla p\rangle_{r}^{2} & \langle\nabla p\rangle_{z}^{2} \\ \langle\nabla p\rangle_{r}^{3} & 0 & 0 & \langle\nabla p\rangle_{z}^{3} & \langle\nabla p\rangle_{\theta}^{3} & 0 \\ 0 & \langle\nabla p\rangle_{z}^{3} & 0 & \langle\nabla p\rangle_{r}^{3} & 0 & \langle\nabla p\rangle_{\theta}^{3} \\ 0 & 0 & \langle\nabla p\rangle_{\theta}^{3} & 0 & \langle\nabla p\rangle_{r}^{3} & \langle\nabla p\rangle_{z}^{3}\end{array}\right]$.

in which the subscripts $(r, z, \theta)$ denote the components and superscripts $(1,2,3)$ the applied pressure drop in the radial, axial and circumferential directions, respectively. The over-determined system is then solved using a least squares approach, in the present case QR-factorisation, to determine the six independent permeabilities. The off-diagonal terms represent the permeability in directions lateral to the applied pressure drop. The permeability tensor can always be expressed as a diagonal tensor, since it is symmetric and positive definite; the tensor is given by,

$\overline{\overline{\boldsymbol{\kappa}}}_{\mathrm{eff}}=\left[\begin{array}{ccc}\lambda_{1} & 0 & 0 \\ 0 & \lambda_{2} & 0 \\ 0 & 0 & \lambda_{3}\end{array}\right]$

in which $\lambda_{1}, \lambda_{2}$ and $\lambda_{3}$ are the eigenvalues that represent the principal permeabilities. To describe the tensor fully, equation 7 comes with three eigenvectors $\mathbf{v}_{\mathbf{1}}, \mathbf{v}_{\mathbf{2}}$ and $\mathbf{v}_{\mathbf{3}}$ which are the principal directions along which transport occurs. 


\section{$2.3 \mathrm{Spectral} / \mathrm{hp}$ element method}

Spatially, equation (4) is solved on a domain that is discretised using the open-source spectral/hp element library nektar ++ (www.nektar.info). As with the finite element method, the domain is tessellated into $N_{e l}$ non-overlapping elements; however, the expansion within each element is high order. Every local physical element can be expressed via an isoparametric coordinated mapping to a standard reference element in which operations such as integration and differentiation are performed. The spectral/hp approach (Karniadakis and Sherwin, 2005) defined over the entire domain can be represented by

$u^{\delta}(\boldsymbol{x})=\sum_{n=1}^{N_{\text {dof }}} \hat{u}_{n} \Phi_{n}(\boldsymbol{x})=\sum_{e=1}^{N_{\mathrm{el}}} \sum_{n=1}^{\operatorname{dim}\left(V^{\delta}\right)} \hat{u}_{n}^{e} \phi_{n}^{e}(\boldsymbol{x})$

where $N_{\text {dof }}$ is the number of degrees of freedom, $\hat{u}_{n}$ are the global expansion coefficients, $\phi_{n}(\boldsymbol{x})$ the polynomials defined in a space $V^{\delta}$ of order $\mathrm{P}$, and $\hat{u}_{n}^{e}$ is the $n^{\text {th }}$ local expansion coefficient within the element $e$. The expansion uses modified Jacobi polynomials in which the interior degrees of freedom are multiplied by linear factors so they are zero on the boundary. This results in natural boundaryinterior decomposition, allowing greater numerical efficiency.

\subsection{High-order splitting scheme}

To solve equation (1), a high-order splitting scheme (operator splitting) is utilised. The scheme is an adaptation of the stiffly-stable velocity-correction projection scheme originally developed for solving the incompressible Navier-Stokes equations (Karniadakis et al., 1991). In this method, the velocity and pressure matrices are decoupled. The Darcy term $\left(\frac{\nu}{\overline{\bar{K}}} \mathbf{u}\right)$ in equation (1) is implemented both implicitly and explicitly into the splitting scheme as follows:

\section{Explicit step}

The first step calculates an intermediate velocity field by:

$\frac{\tilde{\mathbf{u}}-\sum_{q=0}^{J_{i}-1} \frac{\alpha_{q}}{\gamma_{0}} \mathbf{u}^{n-q}}{\Delta t}=\nu \overline{\overline{\boldsymbol{\kappa}}}^{-1} \sum_{q=0}^{J_{e}-1} \frac{\beta_{q}}{\gamma_{0}} \mathbf{u}^{n-q}+f^{n+1}$ where the first term on the right hand side represents explicit treatment of the linear Darcy term, the second term represents external forcing, the second $J$ is the integration order and $\alpha_{q}, \gamma_{0}$ and $\beta_{q}$ are the stiffly stable time integration coefficients (Karniadakis and Sherwin, 2005). The values of $\kappa$ may vary at each quadrature point in the domain.

Pressure step

The intermediate velocity field is used to solve a pressure Poisson equation to obtain the pressure at the new time level $(n+1)$ :

$$
\Delta p^{n+1}=\left(\frac{\gamma_{0}}{\Delta t}\right) \nabla \cdot \tilde{\mathbf{u}}
$$

with the consistent boundary condition:

$$
\begin{aligned}
\frac{\partial p}{\partial n}^{n+1}= & -\left[\frac{\partial \mathbf{u}}{\partial t}^{n+1}+\nu \sum_{q=0}^{J-1} \beta_{q}\left(\nabla \times \nabla \times \mathbf{u}^{n-q}\right.\right. \\
& \left.-\nu \overline{\overline{\boldsymbol{\kappa}}}^{-1} \sum_{q=0}^{J-1} \beta_{q} \mathbf{u}^{n-q}\right] \cdot n
\end{aligned}
$$

where the term $\left(\nu \overline{\boldsymbol{\kappa}}^{-1} \sum_{q=0}^{J-1} \beta_{q} \mathbf{u}^{n-q}\right)$ is only activated when an implicit treatment of the Darcy term is enabled.

\section{Viscous step}

From the solution of the pressure, a second intermediate velocity field is calculated:

$$
\tilde{\tilde{\mathbf{u}}}=\tilde{\mathbf{u}}-\left(\frac{\Delta t}{\gamma_{0}}\right) \nabla p^{n+1}
$$

This is used as a forcing term in a Helmholtz problem to obtain the velocity field at the new time level $(\mathrm{n}+1)$

$$
\left(\Delta-\frac{\operatorname{diag}(\overline{\overline{\boldsymbol{\kappa}}})}{\nu}-\frac{\gamma_{0}}{\Delta t \nu}\right) \mathbf{u}^{n+1}=-\left(\frac{\gamma_{0}}{\Delta t \nu}\right) \tilde{\tilde{\mathbf{u}}}
$$

where the term $\left(\frac{\operatorname{diag}(\overline{\bar{\kappa}})}{\nu}\right)$ is only enabled if an implicit treatment of the Darcy term is enabled.

Time discretisation using the splitting scheme leads to three elliptic equations and one pressure Poisson equation, which need to be solved at every time step. Using the spectral/hp element discretisation with a Galerkin approximation, the resulting matrix equation is typically poorly conditioned due to the strong coupling between the different boundary degrees of freedom. For this reason the solver makes use of sub-structuring and low energy preconditioning in order to accelerate the rate of convergence (see Sherwin and Casarin (2001) and Karniadakis and Sherwin (2005) for more details). 
A number of benchmark problems to validate the solver implementation are given in Appendix A.1. The solver also has the ability to include convection, which is important in some applications.

\section{Spatially varying permeability}

For many applications, including transport in the arterial wall, the domain consists of regions of varying permeability, and also impermeable regions. In regions of varying permeability the variability is naturally handled by the Darcy term $\left(\frac{\nu}{\bar{\kappa}} \mathbf{u}\right)$ in equation (1), in which different $\kappa$ values are assigned at each of the quadrature points. For the impermeable regions the Darcy term must be altered to make the region nearly impermeable as discussed in the following section.

\subsection{Fictitious solid obstacles}

To model the portions of the domain that are impermeable it is possible to lower the permeability i.e drive the velocity towards zero. This can be done by adding a penalisation parameter $\varepsilon$ to the Darcy term in equation (1), which alters the permeability in different regions, i.e.

$\frac{\nu \kappa^{-1}}{\varepsilon} \mathbf{u}$

As the value of $\varepsilon$ tends to zero, the velocity also tends to zero and the region becomes nearly impermeable (small $\kappa$ ). Similar ideas have previously been used in non-boundary fitted mesh methods for the incompressible Navier-Stokes equations (Khad et al., 2000), porous media (Hwang and Advani, 2010) and solid mechanics (Düster et al., 2008). A full discussion of various fictitious domain methods for spectral/hp element methods is given by Vos et al. (2008). Other methods have been described in the literature - see for example Liu et al. (2000) and Hansbo and Hansbo (2002). The parameter $\varepsilon$ can be distributed at the quadrature points throughout the domain by the assignment of values depending on whether the region is solid or fluid, as follows:

$$
\begin{aligned}
& \varepsilon=\varepsilon_{\text {fluid }}=1 \\
& \varepsilon=\varepsilon_{\text {solid }} \rightarrow 0
\end{aligned}
$$

Additionally, this parameter can be smoothed across interfaces, which often helps when capturing steep transitions from a fluid to a solid region. In solid regions, care must be taken in choosing the value of $\varepsilon$. In particular, a very low value will lead to a matrix that is ill conditioned (see Schillinger et al. (2012) for a similar methodology in elastodynamics); hence, there is a practical limit on the value of $\varepsilon$ (although low-energy-basis preconditioning gives excellent performance even for low $\varepsilon$ ). The value is also limited by the time-stepping restriction due to the explicit part of the splitting scheme. In the present context, a value of $\varepsilon=0.01$ captures the obstacle sufficiently.

With the present approach, the boundary between solid and fluid is only weakly imposed. A number of approaches have been proposed, such as distributed Lagrange multipliers (Dong et al., 2004), which enforce the boundary between the solid and the fluid exactly. However, for more complicated geometries (see section 3.2) this would require identification of the solid regions, which essentially amounts to segmentation of the geometry; hence, this could be simulated using a boundary fitted approach. An alternative would be using variable polynomial order at the locations of high image intensity gradient to capture these interfaces in greater detail. In the following it will be demonstrated that this level of complexity is not required when assessing mean permeability properties. Further information regarding the merits of the spectral/hp element method for the fictitious approach used in the present study are given in appendix A.2.

\subsubsection{Method validation}

To demonstrate the effectiveness of the methodology, flow around a square object was simulated and compared with the corresponding simulation rafor a meshed obstacle. The geometry is shown in Figure 2 with velocity and pressure overlaid. The domain under consideration had a side of length $l=1$ and a square object in the centre had a side of length $l=0.25$. For the fictitious approach, the parameter $\varepsilon$ was distributed over the domain as $\varepsilon_{\text {fluid }}=1$ and $\varepsilon_{\text {solid }}=0.01$. The permeability of the surrounding fluid was $\kappa=1 \times 10^{-4} \mathrm{~m}^{2}$ and the viscosity $\nu=1$. A pressure drop of $1000 \mathrm{~Pa}$ was applied between the inlet (left) and outlet (right), whilst the no slip condition was used on the upper and lower surfaces. For the reference method the object was meshed exactly. The geometry was discretised using 225 quadrilateral elements with a polynomial order $p=5$. Elements in the vicinity of the square were locally refined. For the fictitious case the geometry was discretised using 1600 elements with a polynomial order $p=3$. Figure 2 demonstrates the resulting velocity and pressure 


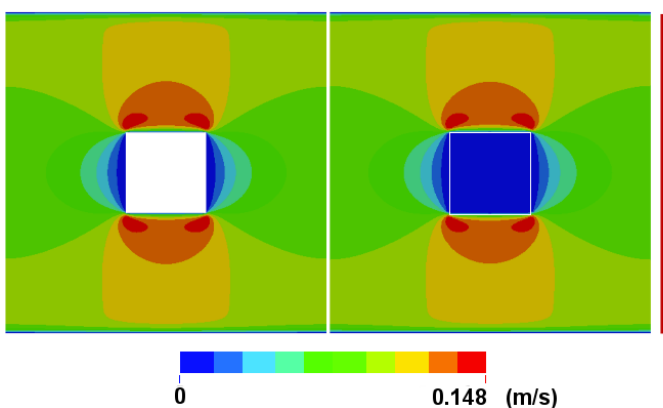

(a) Comparison of velocity contours.

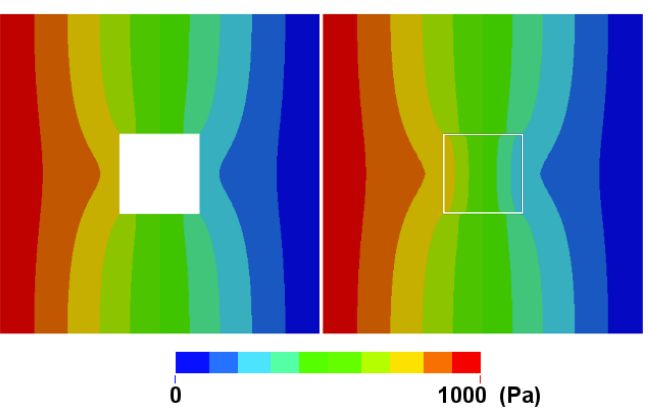

(b) Comparison of pressure contours.

Fig. 2: Comparison of (a) velocity and (b) pressure for different numerical approaches: Meshed geometry (left) and fictitious (right).

fields for the two methods. In the solid region the fictitious approach leads to a velocity field that reduces to a near zero value and the pressure undergoes a linear drop in the streamwise direction. Outside the solid region, the two fields are in good agreement with only small deviations between the methods.

For biological porous media the permeability levels tend to be very low, $O\left(10^{-18} \mathrm{~m}^{2}\right)$. This means that the boundary layer is confined to a very thin layer adjacent to the solid boundary $(O(\sqrt{\kappa}))$. Capturing such a thin boundary layer typically requires high levels of mesh refinement, otherwise non-physica oscillations pollute the solution. This is particularly evident in the solid regions, where small negative values can occur. However, in the present context average properties are of interest; hence only a certain level of accuracy is required.

As a second demonstration, a comparison of effective permeability was performed for flow around a central circular object, which is representative of a SMC and relevant to previous studies of transport in biological porous media (Wang and Tarbell, 1995, Huang and Tarbell, 1997, Dabagh et al., 2009a). The domain under consideration had a side of length $l=1$ and a circular object in the centre which accounted for $20 \%$ of the volume, $\phi=0.2$ (see Figure 3). For the reference method, the geometry was discretised using 180 quadrilateral elements with a polynomial order $p=7$. Elements in the vicinity of the circle were curved and locally refined. For the fictitious case, the geometry was discretised using 1600 elements with a polynomial order $p=5$. To understand the influence of different permeability values, and therefore boundary layer thicknesses, the comparison of methodologies considered two different $\kappa$ values, $\kappa=1 \times 10^{-5}$ and $\kappa=1 \times 10^{-8} \mathrm{~m}^{2}$, with $\nu=1 \mathrm{~m}^{2} / \mathrm{s}$ and $\rho=1$.
For the simulation a pressure drop of $1000 \mathrm{~Pa}$ was applied between the inlet (left) and outlet (right), whilst periodic boundary conditions were used on the upper and lower surfaces.

It is evident from Figure 3 that the results of the two different approaches are qualitatively similar. In the fictitious approach there are some oscillations in the vicinity of the solid object. However Table 1 shows that the permeability obtained using the two methods differs by less than $1 \%$. This demonstrates that the fictitious methodology is effective in determining mean properties of the flow, which is the main aim of the present study. The difference between the methodologies can be further reduced by either $h$ refinement or increasing $p$.

Table 1: Effective permeability obtained by two numerical methods for an array of circles with a volume fraction of $20 \%$. Permeability and effective permeability values are in $\mathrm{m}^{2}$.

\begin{tabular}{cccc}
\hline $\begin{array}{c}\text { Permeability } \\
\left(m^{2}\right)\end{array}$ & $\begin{array}{c}\text { Darcy } \\
\text { Number } \\
\left(k / l^{2}\right)\end{array}$ & $\begin{array}{c}\text { Effective } \\
\text { permeability, } \\
\text { Meshed }\end{array}$ & $\begin{array}{c}\text { Effective } \\
\text { Fictitious }\end{array}$ \\
\hline $1 \times 10^{-5}$ & $3.93 \times 10^{-5}$ & $6.59 \times 10^{-6}$ & $6.66 \times 10^{-6}$ \\
$1 \times 10^{-8}$ & $3.93 \times 10^{-8}$ & $6.66 \times 10^{-9}$ & $6.68 \times 10^{-9}$ \\
\hline
\end{tabular}

To counter the oscillations present for low $\kappa$ values, it is possible to apply a smoothing function to the interface; the abruptness of the change is reduced. A hyperbolic tangent smoothing function was applied across the interface of the solid and fluid, in which a parameter $(\sigma)$ controlled the thickness of the interface. The effective permeability for two values of $\sigma$ is shown in Table 2. For the lower value, the effective permeability is accurate 


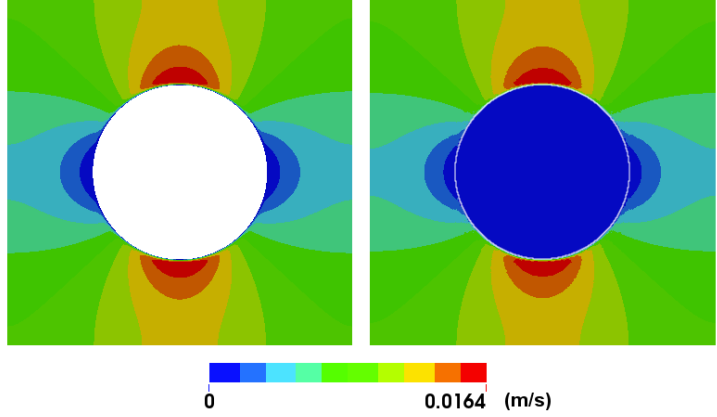

(a) Comparison for $\kappa=1 \times 10^{-5} \mathrm{~m}^{2}$.

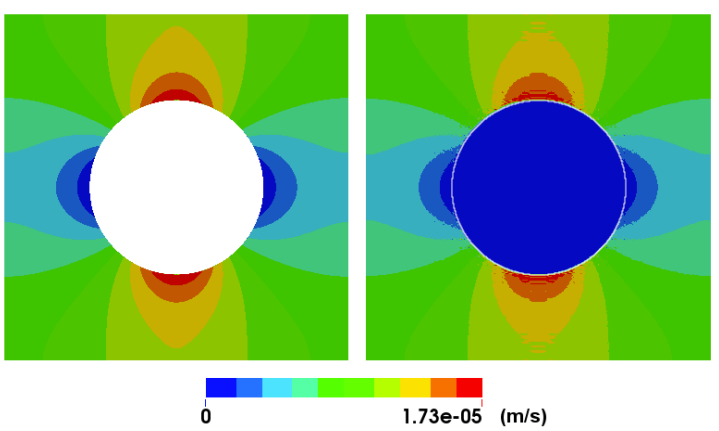

(b) Comparison for $\kappa=1 \times 10^{-8} \mathrm{~m}^{2}$.

Fig. 3: Comparision of velocity for different different numerical approaches and permeabilities. Meshed geometry (left) and fictitious (right).

compared to the $6.66 \times 10^{-9}$ obtained using the exact method (Table 1). However, as $\sigma$ is increased the influence of the smoothing, or more specifically the change in the geometry (the circle is effectively larger), leads to a bigger error. This error could be reduced if the size of the object were decreased. In this study, the fictitious approach without smoothing is preferred as this means the object size is exactly known.

Table 2: Effective permeability of an array of circles with a volume fraction of $20 \%$ and different interface smoothing parameters for the fictitious approach. Permeability and effective permeability values are in $m^{2}$.

\begin{tabular}{cccc}
\hline $\begin{array}{c}\text { Permeability } \\
\left(m^{2}\right)\end{array}$ & $\begin{array}{c}\text { Darcy } \\
\text { Number } \\
\left(\kappa / l^{2}\right)\end{array}$ & $\begin{array}{c}\text { Effective } \\
\text { permeability }\end{array}$ & $\begin{array}{c}\text { Effective } \\
\text { permeability }\end{array}$ \\
& $\sigma=0.01$ & $\sigma=0.04$ \\
\hline $1 \times 10^{-8}$ & $3.93 \times 10^{-8}$ & $6.64 \times 10^{-9}$ & $6.51 \times 10^{-9}$ \\
\hline
\end{tabular}

To make the example more physiological, parameters from previous studies of the medial layer were considered (Wang and Tarbell, 1995, Huang and Tarbell, 1997, Tada and Tarbell, 2000, Dabagh et al., 2009a): SMC volume fraction, $\phi$, of 0.2 and $0.4, \kappa=1.43 \times 10^{-18} \mathrm{~m}^{2} / \mathrm{s}$ (Darcy number: $1.55 \times$ $\left.10^{-7}\right)$. For this case the semi-analytical effective permeability $\left(\kappa_{\text {eff }}\right)$ is given by Wang and Tarbell (1995) as

$\kappa_{\mathrm{eff}}=\kappa \frac{1+\phi-0.305828 \phi^{4}}{1-\phi-0.305828 \phi^{4}}$.

For the numerical simulation, both the meshed and fictitious methods were used. A pressure of 50 $\mathrm{mmHg}$ was applied to one side of the domain and zero pressure to the other. The properties of the interstitial fluid were that of water: kinematic viscosity, $\mu=7.2 \times 10^{-4} \mathrm{~m}^{2} / \mathrm{s}$, density $\rho=998.2 \mathrm{~kg} / \mathrm{m}^{3}$ (Huang and Tarbell, 1997). From Table 3 it is evident that the permeability calculated with the fictitious approach is very accurate for both volume fractions (within $0.66 \%$ of the exactly meshed geometry) and hence we conclude the method is suitable for determining permeability under physiological conditions.

Table 3: Comparison of effective permeability calculated using equation (15), calculated from the meshed circle or using the fictitious approach. The effective permeabilities are $\times 10^{-19} \mathrm{~m}^{2}$.

\begin{tabular}{cccc}
\hline $\begin{array}{c}\text { Volume } \\
\text { fraction }\end{array}$ & $\begin{array}{c}\text { Semi- } \\
\text { analytical }\end{array}$ & $\begin{array}{c}\text { Effective } \\
\text { permeability, } \\
\text { Meshed }\end{array}$ & $\begin{array}{c}\text { Effective } \\
\text { permeability, } \\
\text { Fictitious }\end{array}$ \\
\hline $20 \%$ & 9.53 & 9.52 & 9.52 \\
$40 \%$ & 6.08 & 6.06 & 6.10 \\
\hline
\end{tabular}

\subsection{Permeability of rat arterial media}

In this section, a novel strategy for determining the influence of medial anisotropy on hydraulic permeability is presented. It is based on equilibrating the wall with a fluorescent albumin tracer and then imaging the tracer distribution within the media. The labelled Bovine serum albumin (BSA, $67 \mathrm{kD}$ ) diffuses from the external solution into the wall. It is restricted to the extracellular water space. Clearly, some compartments of this space will be too small for albumin to penetrate, but their small size also means they will have a high resistance 
to flow, and therefore disproportionately little involvement in transmural water flux. For example, the water inside collagen fibrils is not significantly involved in water transport through interstitium and albumin will also be excluded from this space, which has a hydrodynamic radius of only $0.6 \mathrm{~nm}$ (ref to: Levick (1987)). Even if a fixable fluorescent tracer with the same size as water existed, its distribution would not be a useful indicator of the spaces involved in water transport

\section{Fluorescent protein tracer preparation}

The fluorescent dye rhodamine was conjugated to BSA as previously described (Warboys et al., 2010, Nairn and Fothergill, 1986). Briefly, sulforhodamine $\mathrm{B}$ acid chloride (Sigma 86186) in anhydrous acetone was added to $2 \%(\mathrm{w} / \mathrm{v})$ fatty acid free BSA (Sigma, A4612) in carbonate buffer (0.33M, pH9) at $4^{\circ} \mathrm{C}$ with a dye:protein weight ratio of $1: 20$. Unbound dye was removed by gel filtration (Sephadex G-25). The conjugate fraction was dialised against $0.1 \mathrm{X}$ Tyrodes salt solution (TSS; $0.8 \mathrm{~g} / 1 \mathrm{NaCl}, 20 \mathrm{mg} / 1$ $\mathrm{KCl}, 20 \mathrm{mg} / \mathrm{l} \mathrm{CaCl}{ }_{2}, 10 \mathrm{mg} / 1 \mathrm{MgCl}_{2}, 5 \mathrm{mg} / 1 \mathrm{NaH}_{2} \mathrm{PO}_{4}$, $5 \mathrm{mg} / 1 \mathrm{Na}_{2} \mathrm{HPO}_{4}, \mathrm{pH} 6.5$ ), snap frozen drop-wise in liquid nitrogen, lyophilised and stored at $-20^{\circ} \mathrm{C}$. Immediately prior to use, the conjugate was reconstituted in distilled water and 1X TSS to obtain a final rhodamine albumin concentration of $10 \mathrm{mg} / \mathrm{ml}$ in $1 \mathrm{X}$ TSS. It was supplemented with $1 \mathrm{~g} / \mathrm{l}$ glucose, $30 \mathrm{mg} / \mathrm{ml}$ unlabelled BSA, $100 \mathrm{U} / \mathrm{ml}$ penicillin, $100 \mu \mathrm{g} / \mathrm{ml}$ streptomycin, $2.5 \mu \mathrm{g} / \mathrm{ml}$ amphotericin and $50 \mu \mathrm{g} / \mathrm{ml}$ gentamycin. Any remaining free dye was removed by stirring with charcoal, followed by centrifugation $(3000 \mathrm{~g}, 2 \times 15 \mathrm{~min})$ and filtration $(0.2 \mu \mathrm{m})$ to remove the charcoal.

\section{Animal procedures}

Male Sprague-Dawley rats (300-350g) were given 1000U heparin ip 10 minutes before sacrifice by overdose of pentobarbitone. Their aortic bifurcations were excised, cleaned of surrounding fatty tissue, and incubated for $6 \mathrm{~h}$ at $37^{\circ} \mathrm{C}, 5 \% \mathrm{CO}_{2}$ in the tracer solution. After incubation, the bifurcation was placed in fresh TSS and the lumen was flushed with $10 \mathrm{ml}$ TSS to remove any remaining tracer solution. The rhodamine albumin in the tissue was fixed by perfusing the lumen with $10 \mathrm{ml}$ $15 \%$ neutral-buffered formaldehyde (NBF) followed by immersion in $15 \% \mathrm{NBF}$ for $24 \mathrm{~h}$. The same tracer and fixation protocol have been used in our in vivo experiments (Clarke et al., 2012), which gave values of arterial mass transfer coefficients (c. $10^{-8}$ $\mathrm{cm} / \mathrm{s}$ ) in good agreement with those previously found by many unrelated techniques.

\section{Epoxy embedding}

Samples were dehydrated through an ethanol series (10 $\min$ in 50\%, 70\%, 90\%, 95\%; 3x 10 min 100\%; $2 \mathrm{~h}$ in $100 \%$ ) and over night in $100 \%$ propylene oxide (PO). Infiltration in epoxy resin (TAAB; $44 \%$ Embed 812, 35\% dodecenylsuccinic anhydride, $18 \%$ methyl nadic anhydride, $3 \%$ benzyldimethylamine) was performed gradually: 1:1 epoxy/PO, 12h; 2:1 epoxy/PO, 12h; $100 \%$ epoxy, 24h. Curing was carried out in fresh $100 \%$ epoxy at $65^{\circ} \mathrm{C}$ for $48 \mathrm{~h}$. Samples were cut in the plane of all three vessels and the cut face was polished with wet/dry polishing papers (2000, 4000, 6000 grit) using water as a lubricant.

\section{Imaging}

Samples were imaged on an inverted laser scanning confocal microscope (Leica, TCS SP5) with a $\times 40$, $1.25 \mathrm{NA}$ oil immersion lens. Excitation and emission wavelengths were 458/495-555nm and 575/585$620 \mathrm{~nm}$ for autofluorescence and rhodamine fluorescence respectively. Voxel size was: $\mathrm{x}=261.2 \mathrm{~nm}$; $\mathrm{y}=261.2 \mathrm{~nm} ; \mathrm{z}=167.8 \mathrm{~nm}$ (radial, axial and circumferential directions, respectively). Recorded fluorescence intensities were normalised in each z-slice to achieve saturation of $0.4 \%$ of the pixels. This corrected for intensity attenuation with depth.

\section{Equilibrium distribution}

We determined whether the concentration of the tracer in tissue had reached an equilibrium in these experiments by comparing intensity profiles through the wall after different durations of incubation: $3 \mathrm{~h}$, $6 \mathrm{~h}$ and $24 \mathrm{~h}$. The results demonstrated that $6 \mathrm{~h}$ was sufficient (data not shown).

\section{Intensity extraction to quadrature points}

To extract the confocal data to the quadrature points of the spectral/hp expansion, thus providing both a geometric representation and the relative permeability of different tissue components in the media, the following steps were performed:

- Beginning with a simple computational mesh, a spectral/hp expansion (see Figure 4) was created and the polynomial expansion coefficients were transformed to the physical values at the quadrature points. This allowed information to 


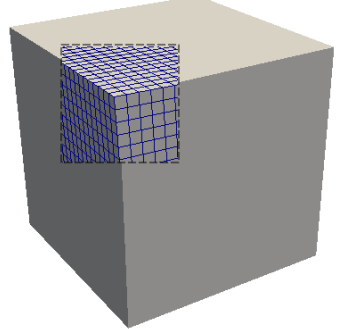

(a) Computational domain with inset showing quadrature points overlaid.

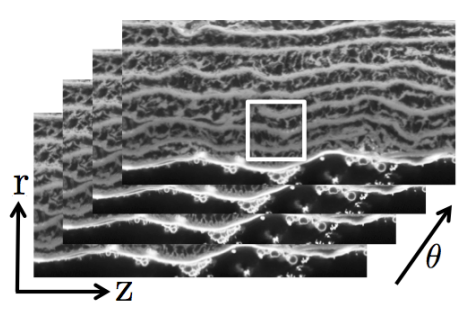

(b) Example image stack on which racer intensity is sampled. White box represents example location where intensity is sampled.

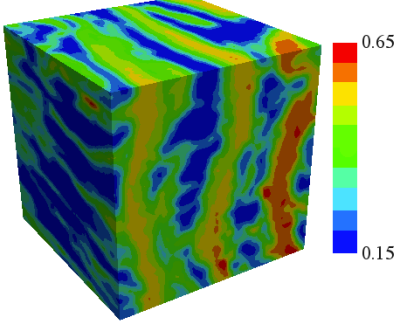

(c) Example computational domain with normalised intensity image data at the quadrature points

Fig. 4: Sequence of steps to transfer image data to the quadrature points.

be accessed and written to the quadrature points of the domain (see Figure 4(a)).

- Using the open-source library VTK (http:// www.vtk.org), the confocal image stack was imported and mapped onto the same space as the quadrature points (see Figure 4(b)). The intensity values from the image were sampled at each quadrature point of the expansion and an intensity (normalised by maximum image intensity) was assigned to each quadrature point. The final normalised intensity distribution extracted onto the computational mesh is illustrated in Figure 4(c).

- The physical values were then forward transformed from the values at the quadrature points to the coefficients. This allowed the expansion to be read onto any points distribution when solving the transport equations. In particular, increased quadrature orders could be utilised to capture steep transport gradients.

Images of tracer intensity were captured using a high-power oil immersion lens and small voxel dimensions; the model therefore captures inhomogeneities and anisotropies in transport properties of the extracellular space with sub-micron resolution.

\section{Medial structure}

Eight medial blocks were extracted from the confocal data, four being located in the inner media (1) - (4) in Figure 6) and four in the outer media (5) - 8 in Figure 6). One block of each type was obtained from each axial position; thus (1) and (5) represent the inner and outer medial blocks, respectively, at one axial location, and so on. In Figure 6, three distinct components are evident: blue areas which represent the borders of SMCs, from which tracer was excluded; yellow/red areas which are the elastic lamellae; and the green areas in between which represent a matrix of elastin, collagen and glycosaminoglycans. The most noticeable difference between the inner and outer media is in the proportion of lamellae; mean volume fractions of lamellae are 0.24 and 0.29 , respectively. The SMC volume fractions remain relatively consistent through all these regions.

To determine the accuracy of this method, three different levels of $h$ refinement (reducing the characteristic local mesh size) were utilised, $h=0.1$, 0.05 and 0.025 , which, with polynomial order $p=$ 3 , correspond to $125000,1 \times 10^{6}$ and $8 \times 10^{6}$ sampling points. This polynomial order was found to be a good balance between computational efficiency and solution accuracy for capturing the thin velocity boundary layer in simulations than a larger characteristic mesh size or higher polynomial order; hence, it was used in all subsequent simulations. At the smallest $h$ a higher polynomial order did improve the solution (reduced oscillations), but $p=3$ was considered sufficiently refined.

The accuracy of the confocal data reconstruction was assessed by comparing it with the intensity distribution of the confocal image data. To do this, a line through the centre of the confocal data $(x=0.5, y=0.5$ and $0 \leq z \leq 1)$ was plotted against the extracted quadrature point data at the same location. Figure 5 demonstrates that below $h=0.05$ (solid black line and crosses) the confocal data (grey line) is captured to a reasonable degree.

From the normalised intensity values $\left(I_{q}\right)$, which are distributed at the quadrature points, it is possible to assign permeability values for different struc- 


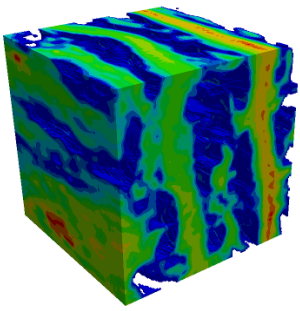

(1)

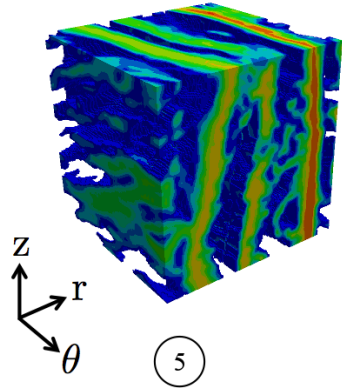

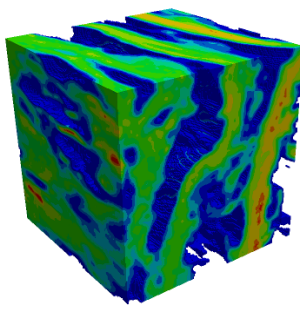

(2)

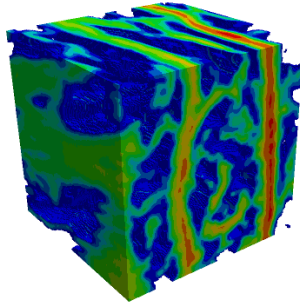

(6)

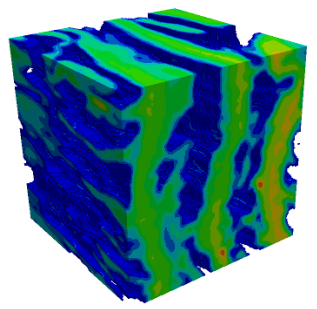

(3)

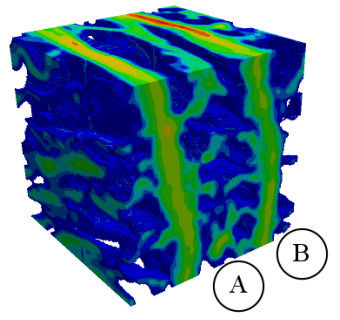

(7)

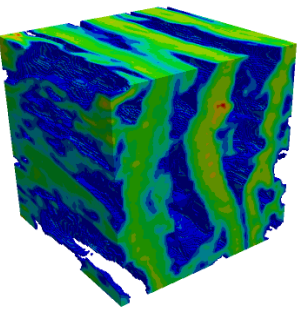

(4)

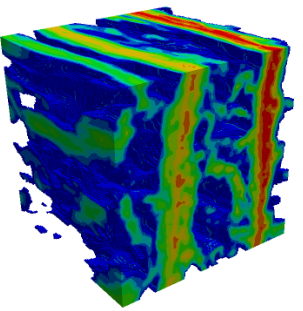

(8)

Fig. 6: Medial blocks $(26.1 \times 26.1 \mu \mathrm{m})$ with SMCs removed at four different axial locations corresponding to the inner media (1), 2, (3) and (4)) and the outer media (5, (6), (7) and (8)).

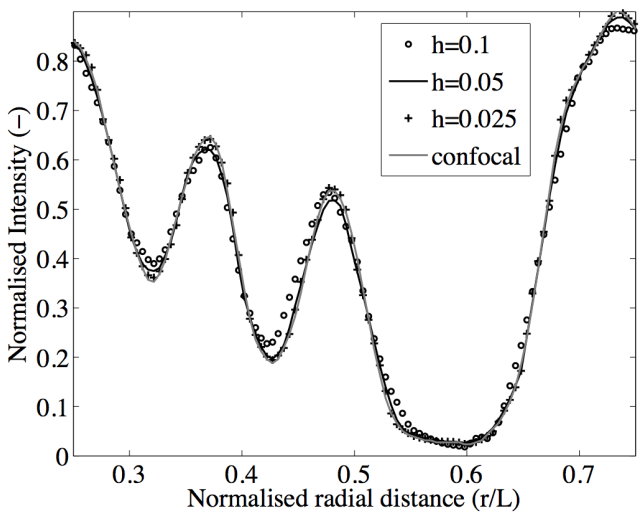

Fig. 5: Comparison of intensity values at the quadrature points with the confocal intensity values. Radial line extracted from the centre of each data set for different levels of h-refinement and zoomed to a central region to show differences. $\mathrm{L}$ $=26.1 \mu \mathrm{m}$

tures of the medial layer. In the present study, SMC content was determined by thresholding the confocal images at the intensity value that corresponded to the border of the cells $\left(I_{s m c}\right)$. The elastic lamellae were identified next, again by thresholding the confocal images but this time at the intensity value that corresponded to the border of the lamellae $\left(I_{\text {lam }}\right)$. Using this method the SMC volume fraction in the GS was consistently around $40 \%$. The permeability of the GS for rabbit medial tissue is in the range $1.3-1.43 \times 10^{-18} \mathrm{~m}^{2}$ (Dabagh et al., 2009a, Huang and Tarbell, 1997); hence, although the permeability in the GS is spatially varying, the mean value in the region above $I_{s m c}$ but below $I_{\text {lam }}$ should be in this range. The permeability at each quadrature $\left(\kappa_{q}\right)$ point in the block was assigned relative to this mean using the following formula:

$\kappa_{q}=\frac{1.32 \times 10^{-18}}{I_{m}} I_{q} \quad m^{2}$

Thus different geometries were given the same mean permeabilities despite having different values of mean GS intensity $\left(I_{m}\right)$. The permeability of regions with intensity above $I_{\text {lam }}$ was also assigned using equation 16, thus setting the lamellar permeability relative to the mean GS permeability. Permeability in the SMCs $\left(I_{q}<I_{s m c}\right)$ was reduced by a factor of 100 , as discussed above. 
Table 4: Principal tensor components for medial block 7 for different levels of $h$ and $p$ refinement.

\begin{tabular}{ccccc}
\hline $\mathrm{h}$ & 0.1 & 0.05 & 0.025 & 0.025 \\
$\mathrm{p}$ & 3 & 3 & 3 & 5 \\
\hline$\lambda_{1}$ & 9.97 & 10.33 & 10.60 & 10.64 \\
$\lambda_{2}$ & 8.25 & 8.48 & 8.71 & 8.74 \\
$\lambda_{3}$ & 4.72 & 5.19 & 5.51 & 5.64 \\
\hline
\end{tabular}

\section{Convergence}

The accuracy of the fictitious approach at high Darcy number (i.e. low permeability) was assessed by performing simulations for medial block 7 in Figure 6 with variable mesh density ( $h=0.1,0.05$ and 0.025$)$ and polynomial order $(p=3,5)$. The block was subject to a pressure drop of $26.1 \mathrm{mmHg}$ in each of the coordinate directions which, given the thickness of the rat arterial wall, corresponded to a transmural pressure drop of $100 \mathrm{mmHg}$. Zero flow conditions were specified on the upper, lower and side surfaces of the cube (Durlofsky, 2005). Table 4 illustrates the principal permeability components for four different cases. The largest $h$ value shows poor accuracy; this is primarily attributed to the less accurate confocal data reconstruction (see Figure 5 ). The much smaller change between $h=0.025, p=3$ and $h=0.025, p=5$ suggests that the former resolution is sufficiently refined and can be considered converged both geometrically and fluid mechanically.

\section{Results}

Figure 7 demonstrates the velocity and pressure distribution in a representative block of medial tissue. In each case a pressure drop of $26.1 \mathrm{mmHg}$ was applied to the tissue in the main coordinate directions and the resulting velocity field was extracted. From this velocity and pressure field the permeability of the structure was determined using equation 6.

Table 5 shows the porosities and the principal permeability components for each medial block in Figure 6 (locations (1) - 8)). If the full permeability tensor is calculated for the above cases, the off-diagonal directions are an order of magnitude smaller and mainly comprise some lateral transport caused by variations in SMC density and alignment. In all blocks the permeability was lowest in the radial direction $\left(\lambda_{1}\right)$ and highest in the circumferential direction $\left(\lambda_{3}\right)^{1}$. Additionally, the radial permeability component appears to be lower in the inner media (compared to the corresponding location in the outer media) for cases (1) - (3). At location (4) this trend is reversed (see section 5 for details)

Table 6 details the principal permeability components for block (7) for three different SMC volume fractions; each column of the table represents the permeability in one principal direction for different SMC volume fractions. In all cases the transport was most favoured in the circumferential direction (along SMCs), with a drop in the axial and radial directions. The permeability of the media reduced as the SMC volume fraction increased, which is to be expected as transport channels between the SMCs will be narrower.

Table 6: Principal tensor components calculated for a $26.1 \mu m \times 26.1 \mu m$ block of outer media (location 7 in Figure 6 ) for varying SMC volume fraction. Components represent the permeability in the principal coordinate directions. Mean principal vectors for the three eigenvalues: $\mathbf{v}_{\mathbf{1}}=[0.01,0.13,0.99], \mathbf{v}_{\mathbf{2}}=[-0.17,0.98,-0.13]$ and $\mathbf{v}_{\mathbf{3}}=[0.99,0.17,-0.03]$. The tensor entries are $\times 10^{-19} \mathrm{~m}^{2}$. ${ }^{*}$ Actual SMC volume fraction of region.

\begin{tabular}{cccc}
\hline SMC volume fraction & $\lambda_{1}$ & $\lambda_{2}$ & $\lambda_{3}$ \\
\hline 0.2 & 13.19 & 11.70 & 9.65 \\
0.3 & 11.93 & 10.14 & 7.57 \\
$0.404^{*}$ & 10.60 & 8.71 & 5.51 \\
\hline
\end{tabular}

The effective permeability of media devoid of elastic lamellae is another area of interest. For this reason, a block of tissue $(6.5 \mu m \times 26.1 \mu m \times$ $26.1 \mu \mathrm{m}$ ) was extracted from the region between the two elastic lamellae (The radial thickness was centred around location (A) in Figure 6). The principal effective permeability components of this tissue block were then calculated for the three different SMC volume fractions (same values as given in table 6$)^{2}$. Table 7 details the principal permeability components obtained from this analysis for the three different SMC volume fractions. Similar trends were observed to those for the whole block (Table 6); however principal transport direc-

1 The principal components can be interpreted as the coordinate direction as the eigenvectors where essentially aligned in these directions.

2 The values obtained with this method have been validated against a larger block of muscular artery to determine that the region is sufficiently large 


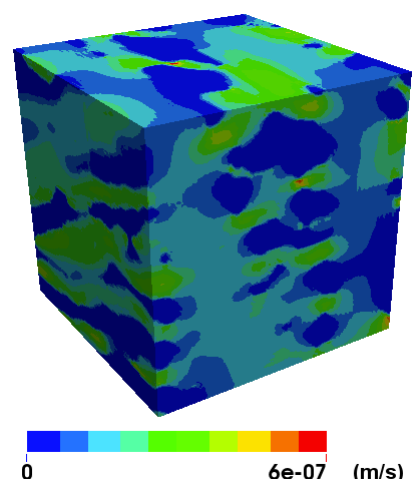

(a) Velocity in the $\mathrm{r}$ (radial) direction.

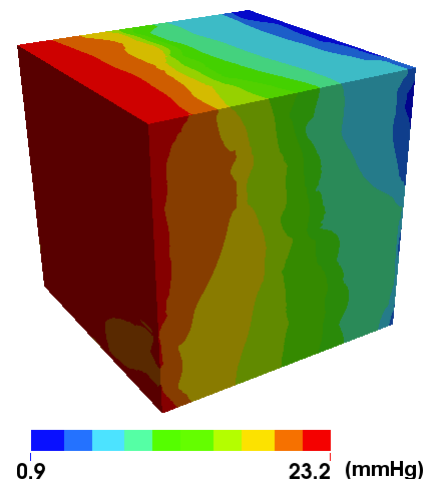

(d) Pressure in the r (radial) direction.

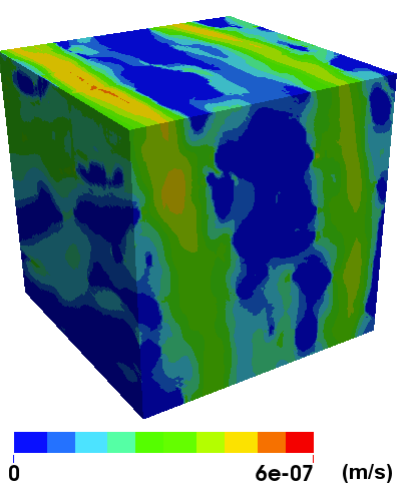

(b) Velocity in the $\mathrm{z}$ (axial) direction.

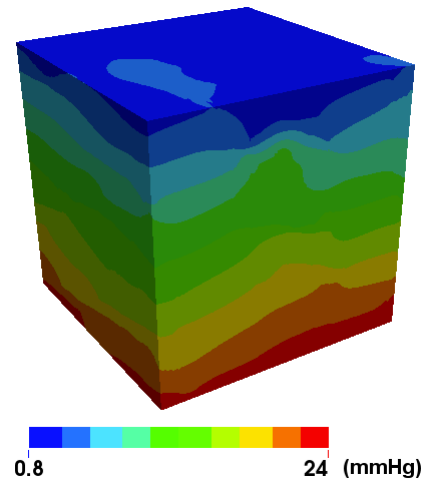

(e) Pressure in the $\mathrm{z}$ (axial) direction.

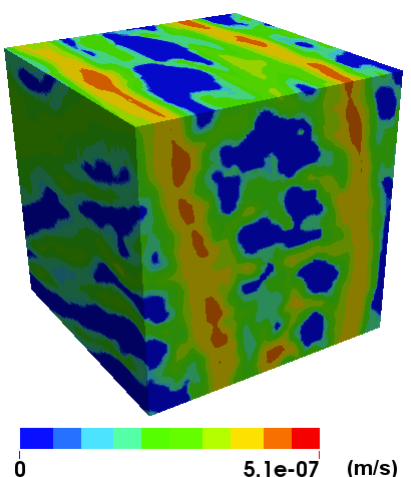

(c) Velocity in the $\theta$ (circumferential) direction.

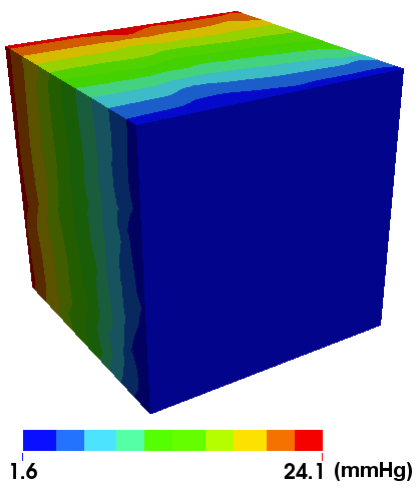

(f) Pressure in the $\theta$ (circumferential) direction.

Fig. 7: Velocity and pressure distributions in medial block (7). In each case the flow direction is indicated by the direction from highest to lowest pressure.

Table 5: Volume fractions and principal tensor components for the eight medial block locations shown in Figure 6.

\begin{tabular}{ccccccccc}
\hline Location & 1 & 2 & 3 & 4 & 5 & 6 & 7 & 8 \\
\hline SMC volume fraction & 0.408 & 0.41 & 0.408 & 0.434 & 0.396 & 0.402 & 0.404 & 0.403 \\
whole volume fraction & 0.30 & 0.303 & 0.293 & 0.292 & 0.30 & 0.308 & 0.303 & 0.3 \\
$\lambda_{1}$ & 10.86 & 12.07 & 10.62 & 10.77 & 11.69 & 10.94 & 10.60 & 13.95 \\
$\lambda_{2}$ & 9.29 & 10.56 & 9.30 & 9.02 & 10.18 & 9.32 & 8.71 & 9.78 \\
$\lambda_{3}$ & 4.12 & 3.44 & 3.95 & 4.92 & 4.61 & 5.14 & 5.51 & 4.01 \\
\hline
\end{tabular}

tions for $\lambda_{2}$ and $\lambda_{3}$ changed for the largest SMC volume fraction. For a SMC volume fraction of 0.2 and 0.3 the principal directions were identical: $\mathbf{v}_{\mathbf{1}}=[0.05,-0.13,0.99], \mathbf{v}_{\mathbf{2}}=[-0.88,0.46,0.1]$ and $\mathbf{v}_{\mathbf{3}}=[0.47,0.88,0.09]$, while for a SMC volume fraction of 0.4 the principal directions were: $\mathbf{v}_{\mathbf{1}}=[0.06,-0.13,0.99], \mathbf{v}_{\mathbf{2}}=[-0.63,0.76,0.14]$ and $\mathbf{v}_{\mathbf{3}}=[0.77,0.63,0.04]$. The change for the latter two eigenvectors was due to the reduction in space between SMCs providing a higher resistance to transport; hence diverting flow in other directions.

Figure 8 shows two planes cut at locations A and $\mathrm{B}$ in Figure 6 to demonstrate the alignment of SMCs within the GS. Between these two interlamellar regions there is a change in the orientation of the SMCs from $-10^{\circ}$ to the circumferential direction to $+45^{\circ}$ to the circumferential direction.

Since the alignment of SMCs changed in the outer media (as shown in Figure 8), an additional 
Table 7: Principal tensor components for a block of GS $(6.1 \mu m \times 26.1 \mu m \times 26.1 \mu m)$, with SMCs, extracted around location (A) in Figure 6). The tensor entries are $\times 10^{-19} \mathrm{~m}^{2}$.

\begin{tabular}{cccc}
\hline SMC volume fraction & $\lambda_{1}$ & $\lambda_{2}$ & $\lambda_{3}$ \\
\hline 0.2 & 11.42 & 9.14 & 8.32 \\
0.3 & 10.17 & 7.24 & 6.53 \\
$0.404^{*}$ & 8.83 & 5.51 & 5.08 \\
\hline
\end{tabular}
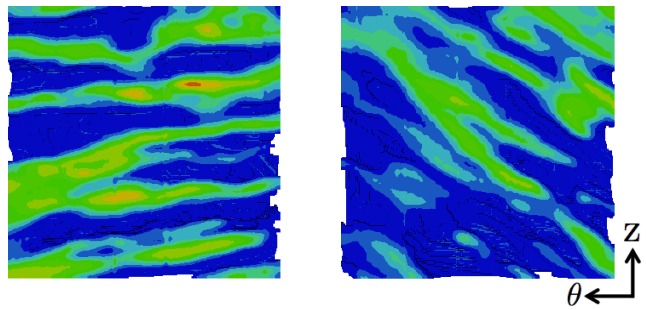

Fig. 8: Slices through the GS for location A (left) and location (B) (right). Locations A and B are indicated in Figure 6.

medial block $(4 \mu m \times 25.6 \mu m \times 26.6 \mu m)$ was extracted around location (B) in Figure 6 to understand the influence of cell orientation. The thickness of the block $(4 \mu \mathrm{m})$ was deemed suitable as tests in location A, which is the thickest layer of SMCs, showed that thickness variations had limited influence on the tensor entries. The full permeability tensor was calculated for this block and the sub-block of GS previously described for Table 7. Table 8 shows the results; circled values represent the entries affected by circumferential cell orientation. For the GS block around location A, the cell orientation promotes transport in the negative $z$ direction for a pressure drop in the $\theta$ direction (see Figure 8 ). This results in a negative tensor entry $\left(\kappa_{z \theta}\right)$. The sign of the transport is due to the right-handed coordinate system. For the GS block around location (B), the cell orientation promotes transport in the positive $z$ direction for a pressure drop in the $\theta$ direction and vice versa (see Figure 8). This results in a positive tensor entry $\left(\kappa_{\theta z}\right)$. The lower magnitude of the values in the second tensor is due to a much higher SMC volume fraction in the GS around location B. If this tensor is diagonalised, the eigenvalues for the tensor at location A are 5.08, 5.51 and 8.83 with respective principal directions $\mathbf{v}_{\mathbf{1}}=$ $[0.77,0.63,0.04], \mathbf{v}_{\mathbf{2}}=[-0.63,0.76,0.14]$ and $\mathbf{v}_{\mathbf{3}}=$ $[0.06,-0.13,0.99]$, and for location (B) are 2.69,
3.09 and 5.78 with respective principal directions $\mathbf{v}_{\mathbf{1}}=[0.25,0.82,-0.51], \mathbf{v}_{\mathbf{2}}=[0.97,-0.18,0.18]$ and $\mathbf{v}_{\mathbf{3}}=[-0.05,0.54,0.84]$. To put this orientation in perspective, for location A the maximum eigenvalue is orientated at $-7.5^{\circ}$ to the circumferential direction, whilst at location B the maximum eigenvalue is orientated at $+32.7^{\circ}$ to the circumferential direction.

Table 8: Effective permeability tensor for two blocks of GS extracted from medial block (7). The first block is $6.1 \mu m \times 26.1 \mu m \times 26.1 \mu m$ extracted around location A in Figure 6. The second block is $4 \mu \mathrm{m} \times 26.1 \mu \mathrm{m} \times 26.1 \mu \mathrm{m})$ extracted around location (B) in Figure 6. The alignment of SMCs is at approximately $-10^{\circ}$ and $+45^{\circ}$ to the circumferential direction for the two locations, respectively. The tensor entries are $\times 10^{-19} \mathrm{~m}^{2}$. The circled entries demonstrate the change in direction due to SMC alignment.

\begin{tabular}{c}
\hline Location \\
\hline A $\quad\left[\begin{array}{ccc}5.27 & -0.24 & 0.17 \\
-0.24 & 5.40 & -0.45 \\
0.17 & -0.45 & 8.77\end{array}\right]$ \\
(B) $\left[\begin{array}{ccc}3.07 & -0.16 & -0.07 \\
-0.53 & 3.60 & 1.39 \\
-0.07 & 1.39 & 4.88\end{array}\right]$
\end{tabular}

\section{Discussion}

The arterial media plays an important role in the regulation of whole wall transport properties (Caro et al., 1980, Lever and Jay, 1990, Lever et al., 1996, Tarbell, 2003) and in particular may contribute to the intimal trapping of macromolecules. Direct measurement of medial permeability, and other regions of the arterial wall for that matter, is difficult, if not impossible; hence, modelling this layer can provide valuable information. In previous models, the medial structure has been treated as either an array of circles (Wang and Tarbell, 1995, Huang and Tarbell, 1997, Tada and Tarbell, 2000, 2002, Dabagh et al., 2009a) or as a homogenous medium with an effective permeability typically derived from the former approaches (Prosi et al., 2005, Ai and Vafai, 2006, Sun et al., 2009, Dabagh et al., 2009b). The array of circles, although useful, 
assumes that the media contains a regular arrangement of SMCs and neglects the influence of the elastic lamellae. The appropriateness of this model for real arteries is unknown (Wang and Tarbell, 1995). The present study introduces an alternative approach for calculating the permeability of the medial tissue. Firstly, the geometry, modelled by the fictitious approach, is realistic, being extracted directly from confocal image data (see section 3.2). Secondly, the relative permeability of the different structures is based on the intensity values from the confocal images (see section 3.2). Finally, the realistic structure can easily be extracted due to the fictitious approach.

The extracted tissue structures (Figure 6) confirm that in this elastic artery the lamellae constitute a large proportion of the volume, with SMCs interspersed. The density of these lamellae are higher at the inner media (locations: (1) - (4)) and reduce in the outer media ((locations: (5) - 8)) Interestingly, the alignment of SMCs differs between different layers of the media. In the inner media the alignment is approximately in the circumferential direction, whilst in the outer media there is notable variation. Figure 8 illustrates the directional change for two different sections (A and $(B$ in Figure 6) of the outer media. SMCs located between the two elastic lamellae (location A) are aligned at approximately $-10^{\circ}$ to the circumferential direction, whilst the cells located at location (B) are rotated by approximately $+45^{\circ}$. The alignment over the outer media tends to alternate slightly between layers; for example, on the left hand side of medial block (7) the SMCs are aligned at $+15^{\circ}$ to the circumferential direction. Different cell orientation in different layers has previously been reported by Clark and Glagov (1985). The SMC density in the GS also shows large variations. This is due to a number of factors. Firstly, the SMCs are not of constant diameter but are spindle shaped; hence regions exist of low SMC volume fraction where one cell finishes and another cell begins. Secondly, there appears to be some level of clustering of the SMCs leading to regions of high and low SMC volume fraction. This is evident at location B in Figure 8(b) and again was noted by Clark and Glagov (1985), who used the term 'fasicles' to describe the clusters.

The permeability results (Table 5) suggest some level of heterogeneity in the arterial wall which is dependent on the region of interest. Consistently through all eight representative medial blocks the permeability is lower radially than in the other two coordinate directions. The mean relative permeability of the eight medial blocks is $1: 2.13: 2.57$, for the radial, axial and circumferential directions, respectively. If sub-blocks of GS with SMCs without lamellae are considered, the ratios of the eigenvalues, are $1: 1.15: 1.84^{3}$. The reduced anisotropy demonstrates that in the representative blocks (locations: (1) - 8) the axial and circumferential permeability is dominated by transport in the lamellae. These observations suggest a mechanism for water to disperse in the non-radial direction, particularly the circumferential direction, as it is transported across the medial layer since the transport in this direction is slightly favoured.

The equivalent isotropic permeability of the media can be expressed by the geometric mean, which gives a measure of the central tendencies:

$\kappa_{\text {mean }}=\sqrt[3]{\lambda_{1} \lambda_{2} \lambda_{3}}$

With the present permeabilities the geometric mean of the eight blocks (Table 5) is $7.86 \times 10^{-19} \mathrm{~m}^{2}$. This number is difficult to compare to literature values as it also includes the influence of the lamellae; these were assumed to be more permeable than the GS. This assumption comes from the experiments: rhodamine fluorescence was highest in the elastic lamellae. It contradicts the widely accepted view that the lamellae are impermeable (Parker and Winlove, 1988). However, previous studies by Katora and Hollis (1975) and Goriely et al. (2007) have demonstrated high permeability of the lamellae to fluorescent albumin tracer and presumably, therefore, to water as well. If the widely accepted view is in fact correct our methods would still be applicable; the relative permeabilities (and hence the detailed results) would be qualitatively similar but would differ in detail. Previous models of the media have reported the permeability of the GS to be around $6.09 \times 10^{-19} \mathrm{~m}^{2}$ (see for example Dabagh et al. (2009b)). For an approximately equivalent SMC volume fraction the principal values for middle blocks of GS without lamellae in locations 5 (8) are: $4.35 \times 10^{-19} \mathrm{~m}^{2}, 5.02 \times 10^{-19} \mathrm{~m}^{2}$ and $8.0 \times$ $10^{-19} \mathrm{~m}^{2}$. If the geometric mean of these permeabilities is considered, the equivalent isotropic permeability of this region would be $5.59 \times 10^{-19} \mathrm{~m}^{2}$, which is close to the literature value. However this value must be interpreted with some caution as the

3 These values are obtained for four outer medial blocks (5 - 8) of SMCs between the lamellae. The size of the blocks varied so they only contained GS and SMCs. As opposed to the whole medial block the principal directions did not align with the coordinate direction 
permeability appears to be location specific (see location (B) in Table 8).

Considering the division of the media into inner (locations (1) - (4)) and outer layers (locations (5) - (8), we observed differences in permeability between these regions. The mean permeabilities of the inner and outer media were $7.57 \times 10^{-19} \mathrm{~m}^{2}$ and $8.14 \times 10^{-19} \mathrm{~m}^{2}$, respectively. The difference was primarily due to a change in the radial permeability. Although the mean permeability was generally lower in the inner layer, the trend was reversed for blocks 4 and 8 . This can be attributed to a number of factors. The inner medial block (location (4)) had a high volume fraction of lamellae (0.335), which in turn led to an elevated radial permeability. Additionally, the arrangement of lamellae resulted in a low resistance pathway to transport radially. The outer medial block (location 8) had low permeability due to a large cluster of SMCs on its left side (visible in Figure 6). This exception highlights the variation that can occur in the arterial wall.

Due to the alignment of SMCs at location B in Figure 8, the permeability tensor (bottom tensor of Table 8) showed larger off-diagonal transport than the equivalent tensor at location A (top tensor of Table 8), indicating that the principal transport directions were different. Additionally the sign of the transport direction changed since the SMCs in the region of interest run from the lower right hand side to the upper left hand side (when looking in the radial direction towards the lumen i.e. the negative $\mathrm{r}$ direction). This directionality is responsible for the change in sign of the circled tensor entries in Table 8 and highlights the complicated transport pathways that exist in the medial layer. Diagonalisation of the tensors in Table 8 demonstrates that the SMC alignment leads to different principal transport directions; maximum eigenvalues were aligned at $-7.5^{\circ}$ and $+32.7^{\circ}$ to the circumferential direction for locations (A) and (B), respectively. However there are some similarities, considering this is a single location, in the level of anisotropy; both regions have a single dominant direction for transport.

The density of smooth muscle cells varied in the medial GS (as evident in Figure 6 and location (B) in Figure 8). This results in variable porosity (for example for the blocks extracted around location A and (B) the SMC volume fraction was 0.4 and 0.54 , respectively); hence, the permeability also varies. Variations in permeability were also observed along a single layer of GS for medial blocks
(5) - (8)). This is due to local changes in density and alignment of SMCs. Overall, these variations demonstrate that the GS is heterogeneous and has regions that both impede and promote the transport of water in different directions. This is a possible mechanism for local accumulation of macromolecules.

\section{Limitations}

The present study has a number of limitations. Firstly, the samples came from one part of the rat aorta. This needs to be extended to other regions of the vessel in order to understand how local structural differences (e.g. at inner versus outer walls of bifurcations) influence local transport properties. Secondly, the samples came from an elastic artery. To obtain a wider description of transport in arterial tissue, samples should also be taken from muscular arteries. Finally, considering other species would provide insight into whether wall transport is species dependent or independent, and hence would indicate the applicability of the present findings to the human aorta.

\section{Conclusion}

A new modelling methodology for determining the permeability of the medial layer of the arterial wall has been presented. Unlike previous approaches, it uses a realistic representation of the structure, obtained from confocal images of the aortic wall, and also allowed the identification of water permeable spaces as well as the relative permeability of wall structures. A fictitious domain approach was used to model the structure, using a newly-developed porous media solver in the spectral/hp element library nektar ++ . The fictitious domain approach was validated by comparison with a basic model of the media, widely used in previous studies. The realistic medial structure affected transport properties; in particular, factors that led to anisotropic transport, characterised in terms of permeability, include: variation in SMC density; different alignments of SMCs; and the elastic lamellae. Overall, the results suggest that transport in the media is spatially varying.

In the future, a wider variety of tissue samples needs to be considered. For example, it will be interesting to determine whether there are different medial permeability properties at different locations within the aortic bifurcation. Finally, the implications for macromolecule transport require investigation. 


\section{Acknowledgements}

This study was funded by the European Commission Marie Curie Integration Fellowship, the British Heart Foundation and the BHF Centre of Research Excellence.

\section{A Appendix}

\section{A.1 Validation of solver}

In the following a number of benchmarks are presented to validate the solver implementation.

\section{$2 D$ forcing}

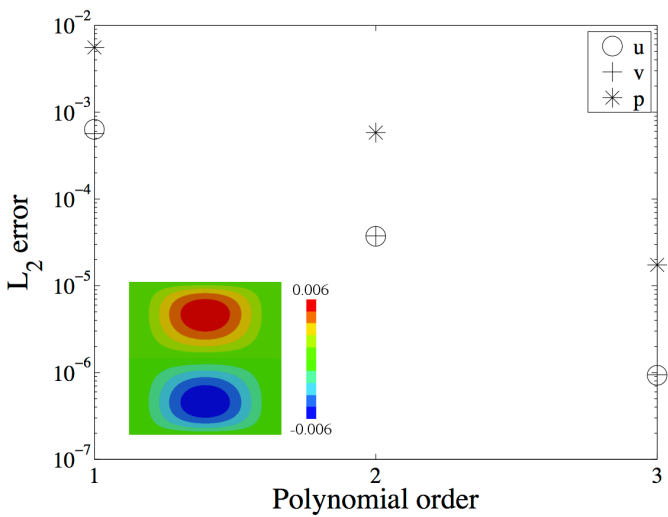

Fig. 9: Results and convergence for the 2D analytical benchmark.

The first benchmark considers the analytical solution given by Masud (2007). For this solution the domain is a $2 \mathrm{D}$ square $([0,1] \times[0,1])$ discretised with 5 quadrilateral elements in each direction. The analytical result for this problem is given by

$$
\begin{aligned}
u(x, y) & =x^{2}(x-1)^{2} y(y-1)(2 y-1), \\
v(x, y) & =-u(y, x), \\
p & =(0.5-x)(0.5-y) .
\end{aligned}
$$

if the domain is subject to a forcing term (f), which is a rearrangement of equation 1 . Figure 9 demonstrates the convergence behaviour for $p=1,2,3$; only these 3 were considered, as the $\mathrm{L}_{2}$ error was extremely small for $p \geq 4$. The $\mathrm{L}_{2}$ error is defined by

$L_{2}$ error $=\left[\int\left(u^{e}(x)-u^{n}(x)\right)^{2} d x\right]^{\frac{1}{2}}$

where $u^{e}(x)$ is the analytical value and $u^{n}(x)$ the numerical value.

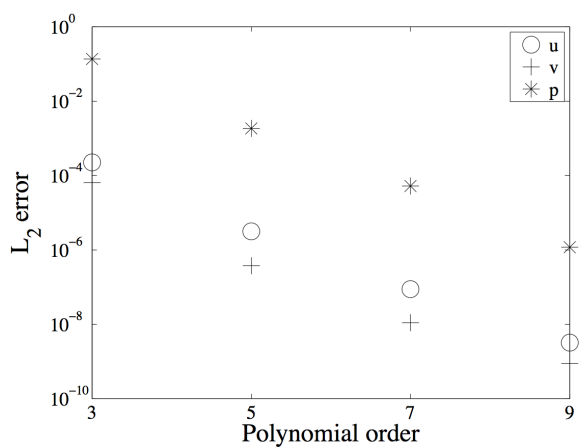

(a) Convergence behaviour, 2D

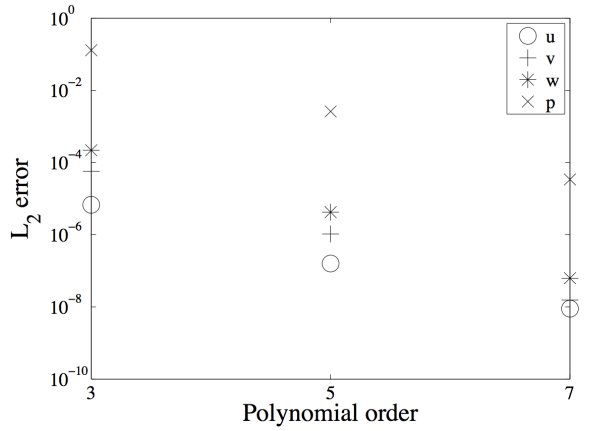

(b) Convergence behaviour, 3D

Fig. 10: Convergence for the channel benchmark.

\section{Porous channel}

Yang et al. (2002) presented a 2D solution of water flow through a channel filled with a polymer gel. The analytical solution to this problem for the velocity and pressure field is given by

$$
\begin{aligned}
w(x, y, z) & =1-\frac{e^{\frac{1-x}{\sqrt{K}}}+e^{\frac{x}{\sqrt{K}}}}{1+e^{\frac{1}{\sqrt{K}}}}, \\
p & =\frac{\nu}{\kappa}(1-z) .
\end{aligned}
$$

This problem demonstrates a particularly challenging feature of biological porous media: very fine boundary layers that exist close to solid objects. To capture such steep boundary layers typically requires very fine meshes. For the numerical solution, a $2 \mathrm{D}$ square $([0,1] \times$ $[0,1])$ was discretised with 96 quadrilateral elements (base element size $h=0.15$ ) and refined in the vicinity of the wall (off-wall spacing $h=0.0125$ ). The convergence behaviour of the numerical solution is demonstrated in Figure 10(a). This highlights a unique advantage of highorder methods, in which small boundary layers can be captured via $p$ refinement; however, as the value of $\kappa$ is lowered the base level mesh size within the boundary layer must be reduced. Equivalent solutions were solved in $3 \mathrm{D}$ and with mixed element types i.e tetrahedral elements in the bulk and prismatic elements in the vicinity of the wall. They demonstrate similar convergence behaviour: convergence for hexahedral based 3D geometry is shown in Figure 10(b). 


\section{A.2 $\mathrm{h}$ versus $\mathrm{p}$ refinement}

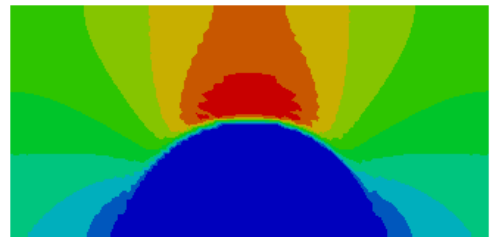

$\mathrm{h}=0.0125, \mathrm{p}=1$ (DOF: 6482)

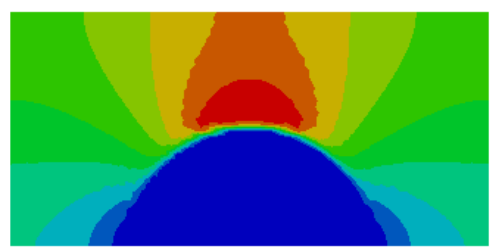

$\mathrm{h}=0.05, \mathrm{p}=4(\mathrm{DOF}:$ 6482)

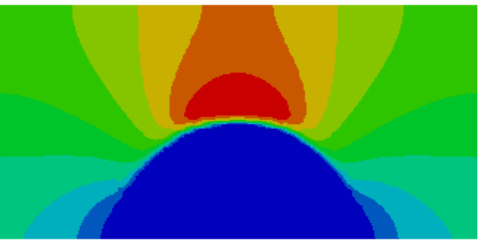

$\mathrm{h}=0.05, \mathrm{p}=5$ (DOF: 10102)

Fig. 11: Contours of velocity around a circular object obtained using the fictitious approach and different levels of $h$ and $p$ refinement. Only half the geometry is shown so that oscillations are more evident.

As a demonstration of the how $h$ and $p$ refinement influences the solution, a square domain with a length $l=1$ and a circular object in the centre accounting for $20 \%$ of its volume, $\phi=0.2$, was simulated. This geometry was chosen as it allowed lower levels of $h$ refinement to be used while demonstrating the advantage of either $h$ or $p$ refinement. Figure 11 shows contours of velocity for different levels of $h$ and $p$ refinement. For the equivalent number of degrees of freedom (DOF) the solution shows fewer oscillations at high $p$ rather than high $h$ (compare $h=0.0125, p=1$ versus $h=0.05, p=4$, top and middle images in 11). As the polynomial order is increased the solution becomes non-oscillatory (bottom image in Figure 11); a greater number of linear DOF (14522) is required to achieve the same accuracy.

To investigate the difference in the solution for the equivalent number of global DOF in more detail, the velocity was extracted along the black line shown in the inset of Figure 12. This line was chosen as it passes through the high velocity region, which is the most difficult part of the solution to capture as a result of the sharp boundary layer that forms around the circular object. Figure 12 shows that the solution for the equivalent linear case exhibits more oscillations than the solution with a lower level of $h$ refinement and higher $p$.

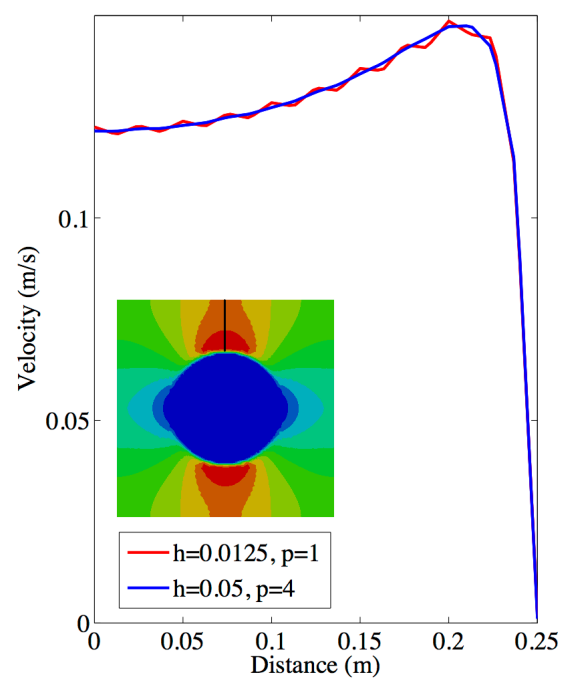

Fig. 12: Comparison of velocity plotted along a line in the high velocity region (see inset) using different combinations of $h$ and $p$ refinement having equivalent degrees of freedom.

\section{References}

L. Ai and K. Vafai. A coupling model for macromolecule transport in a stenosed arterial wall. International journal of heat and mass transfer, 49(9):1568-1591, 2006.

A.Tedgui and M. J. Lever. The interaction of convection and diffusion in the transport of 131i-albumin within the media of the rabbit thoracic aorta. Circulation research, 57(6):856-863, 1985.

F. Bozsak, J-M. Chomaz, and A. I. Barakat. Modeling the transport of drugs eluted from stents: physical phenomena driving drug distribution in the arterial wall. Biomechanics and modeling in mechanobiology, 13(2):327-347, 2014

C. G. Caro, M. J. Lever, Z. Lever-Rudich, F. Meyer, N. Liron, W. Ebel, K. H. Parker, and C. P. Winlove. Net albumin transport across the wall of the rabbit common carotid artery perfused in situ. Atherosclerosis, 37(4):497-511, 1980.

J. M. Clark and S. Glagov. Transmural organization of the arterial media. the lamellar unit revisited. Arteriosclerosis, Thrombosis, and Vascular Biology, 5(1): 19-34, 1985.

L. A. Clarke, Z. Mohri, and P. D. Weinberg. High throughput en face mapping of arterial permeability using tile scanning confocal microscopy. Atherosclerosis, 224(2):417-425, 2012

E. Cutri, P. Zunino, S. Morlacchi, C. Chiastra, and F. Migliavacca. Drug delivery patterns for different stenting techniques in coronary bifurcations: a comparative computational study. Biomechanics and Modeling in Mechanobiology, 12(4):657-669, 2013.

M. Dabagh, P. Jalali, and Y.T Konttinen. The study of wall deformation and flow distribution with transmural pressure by three-dimensional model of thoracic 
aorta wall. Medical Engineering $\mathcal{E}$ Physics, 31(7): 816-824, 2009a.

M. Dabagh, P. Jalali, and J. M. Tarbell. The transport of ldl across the deformable arterial wall: the effect of endothelial cell turnover and intimal deformation under hypertension. American Journal of Physiology-Heart and Circulatory Physiology, 297(3): H983-H996, 2009b.

W. J. Denny, B. M. O'Connell, J. Milroy, and M. T. Walsh. An analysis of three dimensional diffusion in a representative arterial wall mass transport model. Annals of Biomedical Engineering, pages 1062-1073, 2012.

S. Dong, D. Liu, M. R. Maxey, and G. E. Karniadakis. Spectral distributed lagrange multiplier method: algorithm and benchmark tests. Journal of Computational Physics, 195(2):695-717, 2004.

L .J. Durlofsky. Upscaling and gridding of fine scale geological models for flow simulation. In 8th International Forum on Reservoir Simulation Iles Borromees, Stresa, Italy, pages 20-24, 2005.

A. Düster, J. Parvizian, Z. Yang, and E. Rank. The finite cell method for three-dimensional problems of solid mechanics. Computer methods in applied mechanics and engineering, 197(45):3768-3782, 2008.

A. R. Goriely, A. L. Baldwin, and T. W. Secomb. Transient diffusion of albumin in aortic walls: effects of binding to medial elastin layers. American Journal of Physiology-Heart and Circulatory Physiology, 292 (5):H2195-H2201, 2007.

A. Hansbo and P. Hansbo. An unfitted finite element method, based on nitsche's method, for elliptic interface problems. Computer methods in applied mechanics and engineering, 191(47):5537-5552, 2002

T. Y. Hou and X. Wu. A multiscale finite element method for elliptic problems in composite materials and porous media. Journal of computational physics, 134(1):169-189, 1997.

Z.J. Huang and J. M. Tarbell. Numerical simulation of mass transfer in porous media of blood vessel walls. American Journal of Physiology-Heart and Circulatory Physiology, 273(1):H464-H477, 1997.

W.R. Hwang and S. G. Advani. Numerical simulations of stokes-brinkman equations for permeability prediction of dual scale fibrous porous media. Physics of Fluids, 22:113101, 2010.

G. E. Karniadakis and S. J. Sherwin. Spectral $/ h p$ element methods for CFD. Oxford University Press, 2005.

G. E. Karniadakis, M. Israeli, and S. A. Orszag. Highorder splitting methods for the incompressible navierstokes equations. Journal of Computational Physics, 97(2):414-443, 1991.

M. E. Katora and T. M. Hollis. A simple fluorescent method for quantitative determination of aortic protein uptake. Journal of Applied Physiology, 39(1): 145-149, 1975.

K. Khadra, P. Angot, S. Parneix, and J-P. Caltagirone. Fictitious domain approach for numerical modelling of navier-stokes equations. International Journal for Numerical Methods in Fluids, 34(8):651-684, 2000.

M. Khakpour and K. Vafai. Critical assessment of arterial transport models. International Journal of Heat and Mass Transfer, 51(3):807-822, 2008.

A. R.A. Khaled and K. Vafai. The role of porous media in modeling flow and heat transfer in biological tissues. International Journal of Heat and Mass Transfer, 46(26):4989-5003, 2003.
M. J. Lever and M. Jay. Albumin and cr-edta uptake by systemic arteries, veins, and pulmonary artery of rabbit. Arteriosclerosis, Thrombosis, and Vascular Biology, 10(4):551-558, 1990.

M. J. Lever, M. T. Jay, and P. J. Coleman. Plasma protein entry and retention in the vascular wall: possible factors in atherogenesis. Canadian journal of physiology and pharmacology, 74(7):818-823, 1996.

J. R. Levick. Flow through interstitium and other fibrous matrices. Experimental Physiology, 72(4):409 437, 1987.

X. Liu, R. P. Fedkiw, and M. Kang. A boundary condition capturing method for poisson's equation on irregular domains. Journal of Computational Physics, 160(1):151-178, 2000

A. Masud. A stabilized mixed finite element method for darcy-stokes flow. International Journal for Numerical Methods in Fluids, 54(6-8):665-681, 2007.

R. C. Nairn and J. E. Fothergill. Fluorescent protein tracing, Chapter 2. E. \& S. Livingstone Ltd., 1986.

Lars Bo Nielsen. Transfer of low density lipoprotein into the arterial wall and risk of atherosclerosis. Atherosclerosis, 123(1):1-15, 1996.

K.H. Parker and C. P. Winlove. The macromolecular and ultrastructural basis of the permeability properties of the vascular wall. Engineering in medicine, 17 (4):175-180, 1988.

M. Prosi, P. Zunino, K. Perktold, and A. Quarteroni. Mathematical and numerical models for transfer of low-density lipoproteins through the arterial walls: a new methodology for the model set up with applications to the study of disturbed lumenal flow. Journal of biomechanics, 38(4):903-917, 2005.

D. Schillinger, A. Düster, and E. Rank. The hp-dadaptive finite cell method for geometrically nonlinear problems of solid mechanics. International Journal for Numerical Methods in Engineering, 89(9):11711202, 2012.

S. J. Sherwin and M. Casarin. Low-energy basis preconditioning for elliptic substructured solvers based on unstructured spectral/hp element discretization. Journal of Computational Physics, 171(1):394-417, 2001.

N. Sun, R. Torii, N. B. Wood, A. D. Hughes, S. A. Thom, and X. Y. Xu. Computational modeling of ldl and albumin transport in an in vivo ct image-based human right coronary artery. Journal of biomechanical engineering, 131(2):021003-021003, 2009.

S. Tada and J. M. Tarbell. Interstitial flow through the internal elastic lamina affects shear stress on arterial smooth muscle cells. American Journal of Physiology-Heart and Circulatory Physiology, 278(5): H1589-H1597, 2000.

S. Tada and J. M. Tarbell. Flow through internal elastic lamina affects shear stress on smooth muscle cells (3d simulations). American Journal of Physiology-Heart and Circulatory Physiology, 282(2):H576-H584, 2002.

S. Tada and J. M. Tarbell. Internal elastic lamina affects the distribution of macromolecules in the arterial wall: a computational study. American Journal of Physiology-Heart and Circulatory Physiology, 287 (2):H905-H913, 2004

J. M. Tarbell. Mass transport in arteries and the localization of atherosclerosis. Annual review of biomedical engineering, 5(1):79-118, 2003.

J. M. Tarbell. Shear stress and the endothelial transport barrier. Cardiovascular research, 87(2):320-330, 2010 . 
G. Vairo, M. Cioffi, R. Cottone, G. Dubini, and F. Migliavacca. Drug release from coronary eluting stents: a multidomain approach. Journal of biomechanics, 43 (8):1580-1589, 2010.

P. E. J. Vos, R. van Loon, and S. J. Sherwin. A comparison of fictitious domain methods appropriate for spectral/hp element discretisations. Computer Methods in Applied Mechanics and Engineering, 197(25): 2275-2289, 2008.

D. M. Wang and J. M. Tarbell. Modeling interstitial flow in an artery wall allows estimation of wall shear stress on smooth muscle cells. Journal of Biomechanical Engineering, 117:359, 1995.

C. M. Warboys, R. E. Berson, G.E. Mann, J. D. Pearson and P.D. Weinberg. Acute and chronic exposure to shear stress have opposite effects on endothelial permeability to macromolecules. American Journal of Physiology-Heart and Circulatory Physiology, 298(6): H1850-H1856, 2010.

P.D. Weinberg. Rate-limiting steps in the development of atherosclerosis: the response-to-influx theory. Journal of vascular research, 41(1):1-17, 2004.

C. Yang, C. A. Grattoni, A. H. Muggeridge, and R. W. Zimmerman. Flow of water through channels filled with deformable polymer gels. Journal of Colloid and Interface Science, 250(2):466-470, 2002. 